\title{
Manejo de la neumonía del adulto adquirida en la comunidad. Resumen del consenso nacional
}

\author{
Sociedad Chilena de Enfermedades Respiratorias ${ }^{1}$ \\ y Sociedad Chilena de Infectología². \\ Summary of the Consensus for \\ management of community acquired \\ pneumonia in adults
}

This is an update of the Consensus for treatment of community acquired pneumonia in adults, prepared by the Chilean Society of Respiratory Diseases and the Chilean Society of Infectious Diseases. These norms were prepared by thirty specialists in respiratory diseases, internal medicine, infectious diseases, microbiology, intensive medicine and radiology. The purpose of the document is to norm the management of immunocompetent adults with community acquired pneumonia, by the public and private health systems of our country. The complete document will be published in June, in the respective journals of the Societies of Respiratory and Infectious Diseases. This is a summary to obtain a better diffusion of these norms among internists and general practitioners (Rev Méd Chile 2005; 133: 953-67).

(Key Words: Bacterial vaccines; Pneumonia; Respiratory tract infections)

Recibido el 28 de junio de 2005. Aceptado el 4 de julio de 2005.

Coordinador del Consenso: Fernando Saldías $\mathrm{P}^{1}$.

\section{INTRODUCCIÓN}

Fernando Saldías $\mathrm{P}^{1}$, Carlos Pérez $\mathrm{C}^{2}$.

La neumonía adquirida en la comunidad (NAC) es la infección respiratoria aguda que compromete el parénquima pulmonar ocasionada por microorganismos adquiridos fuera del ambiente hospitalario, correspondiendo a un proceso incubado en el

Correspondencia a: Dr. Fernando Saldías Peñafiel. Departamento de Enfermedades Respiratorias, Pontificia Universidad Católica de Chile. Marcoleta 345, 4o piso. Santiago, Chile. Teléfonos: 6331541-3543242. Fax: 6335255. E mail: fsaldias@med.puc.cl ambiente comunitario $^{1}$. La gravedad de la neumonía puede variar desde un cuadro banal, en sujetos jóvenes previamente sanos, que puede confundirse con infecciones del tracto respiratorio superior, bronquitis o gripe, hasta cuadros graves que deben ser manejados en unidades especializadas de cuidado intensivo y ponen en peligro la vida de los pacientes. Los principales mecanismos de adquisición de una neumonía son la aspiración de contenido bucofaríngeo o gástrico, la inhalación de aerosoles (virus, Legionella sp, Mycobacterium tuberculosis), la diseminación hematógena de otro foco infeccioso, la iatrogénica (por instrumentalización de la vía aérea o espacio pleural) y la infección por vecindad. 
En nuestro país, la neumonía es la principal causa de muerte por enfermedades infecciosas y la tercera causa específica de muerte en la población adulta ${ }^{2,3}$. Reconociendo la importancia de la NAC, numerosos países han elaborado guías clínicas con el propósito de racionalizar el manejo de los enfermos, siendo las más conocidas en el medio nacional las guías clínicas de la Sociedad Americana de Tórax (ATS) ${ }^{4}$, la Sociedad Británica de Tórax (BTS $)^{5}$ y la Sociedad Americana de Enfermedades Infecciosas (IDSA) ${ }^{6}$. En 1999, un comité de expertos de la Sociedad Chilena de Enfermedades Respiratorias elaboró la primera guía nacional de manejo de la neumonía comunitaria del adulto, basado fundamentalmente en la experiencia extranjera adaptada a la organización del sistema de salud de nuestro país 7 . En la última década, han ocurrido cambios epidemiológicos y tecnológicos significativos, que han obligado a las sociedades científicas internacionales a actualizar las guías clínicas de neumonía, entre los que cabe mencionar la identificación de nuevos patógenos respiratorios involucrados en la neumonía del adulto (Mycoplasma pneumoniae, Chlamydia pneumoniae, Legionella pneumophila); el aumento de la población senescente con comorbilidad múltiple; la emergencia de patógenos respiratorios resistentes a los antimicrobianos asociado al uso indiscriminado de antibióticos (las tasas de resistencia de $\mathrm{S}$ pneumoniae a penicilina fluctúan entre $20-30 \%$ y a macrólidos entre $10-15 \%$ ); el desarrollo de nuevos antimicrobianos por la industria farmacéutica, efectivos para el tratamiento de la neumonía (macrólidos, ketólidos y fluoroquinolonas); y el desarrollo de nuevas técnicas de detección de antígenos, serología y biología molecular para el diagnóstico de las infecciones respiratorias. Basados en estos antecedentes, un comité de expertos de la Sociedad Chilena de Enfermedades Respiratorias y la Sociedad Chilena de Infectología han revisado la evidencia nacional y extranjera concernientes con el manejo de la neumonía adquirida en la comunidad del adulto, previo a elaborar las actuales recomendaciones clínicas que tienen por objetivo ser aplicadas en los sistemas público y privado del medio nacional.

\section{EPIDEMIOLOGÍA}

Gonzalo Valdivia $C^{1}$.

Las enfermedades respiratorias constituyen la tercera causa de muerte de la población chilena, superadas sólo por las enfermedades del aparato circulatorio y los tumores malignos ${ }^{2}$. El 50\% de los decesos por enfermedades respiratorias en el adulto son atribuibles a neumonía, elevándose sobre $80 \%$ en la población pediátrica. La neumonía es la principal causa de muerte por enfermedades infecciosas en la población chilena y la primera causa específica de muerte en los mayores de 80 años $^{3}$. La incidencia y letalidad de la neumonía comunitaria se elevan en las edades extremas de la vida (menores de un año y mayores de 65 años). Así, la tasa de mortalidad por neumonía en la población senescente alcanza a 6,6 muertes por cada 1.000 personas.

En los estudios poblacionales se ha estimado que cerca de $80 \%$ de los pacientes con NAC pueden ser manejados en el ámbito ambulatorio (consultorios y Servicios de Ungencia) debido a su bajo riesgo de complicaciones y muerte (letalidad inferior a $1-2 \%$ ), y menos de $20 \%$ de los episodios deben ser admitidos al hospital debido a la gravedad de la infección pulmonar, concentrándose en esta población el mayor riesgo de complicaciones, muerte y demanda de recursos de salud ${ }^{8}$. El costo económico de la NAC manejada en el hospital es veinte veces superior a aquella tratada en el ámbito ambulatorio.

En nuestro país, la mortalidad por neumonía ha declinado significativamente a partir de 1999 (46,4 muertes por 100.000 habitantes), lo cual puede ser atribuido a una mejor distribución de los recursos humanos y materiales durante la campaña de invierno, mayor accesibilidad a los servicios de salud y oportunidades de tratamiento de la población ${ }^{3}$. La incidencia y mortalidad de la neumonía tienen un patrón estacional en nuestro país, concentrándose en los meses de otoñoinvierno. Similar a lo acontecido en otros países, las tasas de letalidad por neumonía comunitaria del adulto reportadas en nuestro medio pueden variar ampliamente entre los distintos Servicios de Salud.

La edad avanzada (mayores de 65 años), la presencia de comorbilidad, el consumo de tabaco, el alcoholismo y la contaminación ambiental 
fuera y dentro del hogar constituyen factores de riesgo independientes de adquirir una neumonía comunitaria y se cree que su elevada prevalencia en países como el nuestro explica, en parte, la situación de la mortalidad por neumonía.

Los costos del cuidado de esta enfermedad han aumentado progresivamente, concentrándose en los pacientes hospitalizados y admitidos a unidades de cuidado crítico por NAC grave ${ }^{8,9}$. Considerando que estamos en presencia de una patología infecciosa potencialmente prevenible, cuya historia natural es conocida, que tiene tratamiento específico y determina un quiebre en la calidad de vida de los enfermos, especialmente en las edades extremas de la vida; la neumonía comunitaria debe ser considerada como un grave problema de salud pública.

\section{Diagnóstico CLínico-RAdiográfico}

Rodrigo Gil D ${ }^{1}$, Patricia Fernández V1, Eduardo Sabbagh $\mathrm{P}^{1}$.

La neumonía comunitaria del adulto es un cuadro de evolución aguda, caracterizado por compromiso del estado general, fiebre, calofríos, tos, expectoración purulenta y dificultad respiratoria de magnitud variable; asociado en el examen físico a alteración de los signos vitales (taquicardia, taquipnea, fiebre) y signos focales en el examen pulmonar: matidez, disminución del murmullo pulmonar, crepitaciones, broncofonía y egofonía. Sin embargo, la probabilidad de un paciente con síntomas respiratorios agudos de tener una neumonía depende de la prevalencia de la enfermedad en el ambiente donde se presenta y de las manifestaciones clínicas del enfermo. Así, se ha estimado que la prevalencia de neumonía en los servicios de atención ambulatoria (consultorios y Servicios de Urgencia) no supera el 3-5\% de las consultas por patología respiratoria ${ }^{10}$.

El diagnóstico clínico de neumonía sin confirmación radiográfica carece de precisión, ya que el cuadro clínico (historia y examen físico) no permite diferenciar con certeza al paciente con neumonía de otras condiciones respiratorias agudas (infecciones de la vía aérea superior, bronquitis, influenza) ${ }^{11}$. El diagnóstico de neumonía basado exclusivamente en criterios clínicos tam- bién se ve dificultado por la gran variabilidad en la capacidad de detectar signos focales en el examen de tórax entre distintos observadores.

El adulto mayor ( $>65$ años) suele tener neumonías de presentación atípica, que dificultan el diagnóstico y retrasan el inicio del tratamiento, afectando adversamente el pronóstico de los enfermos $^{12}$. Estos pacientes suelen no presentar los síntomas respiratorios clásicos o fiebre, consultando por síntomas inespecíficos tales como decaimiento, rechazo alimentario, somnolencia o confusión mental, o por descompensación de enfermedades crónicas.

El diagnóstico de la neumonía comunitaria del adulto es clínico-radiográfico: la historia y examen físico sugieren la presencia de una infección del tracto respiratorio inferior, pero el diagnóstico de certeza se establece cuando se demuestra la aparición reciente de infiltrados pulmonares en la radiografía de tórax.

A los médicos de atención primaria se les recomienda solicitar radiografía de tórax en las siguientes circunstancias clínicas: a) paciente que consulta por tos, expectoración, fiebre o dificultad respiratoria de evolución aguda, y presenta algún signo focal en el examen pulmonar; b) adulto mayor de 65 años con compromiso de conciencia o descompensación de una enfermedad crónica de causa desconocida; c) paciente portador de enfermedad cardiovascular o respiratoria crónica (cardiopatía isquémica, insuficiencia cardíaca congestiva, EPOC, bronquiectasias), que consulta por tos, expectoración o fiebre, independientemente de los hallazgos en el examen pulmonar. En general, si un paciente consulta por tos o expectoración y no tiene alteraciones de los signos vitales, el estado de conciencia y el examen pulmonar, se recomienda no solicitar una radiografía de tórax, ya que la probabilidad de neumonía en estas circunstancias es muy baja ${ }^{13}$.

La radiografía de tórax frontal y lateral debe ser uno de los exámenes de rutina en el diagnóstico y evaluación de los pacientes con neumonía comunitaria. La radiografía permite confirmar el diagnóstico clínico, establecer su localización, extensión y gravedad; además permite diferenciar la neumonía de otras condiciones agudas (insuficiencia cardíaca congestiva, tromboembolismo pulmonar, neumotórax, daño pulmonar por drogas o de origen inmunológico), detectar posibles 
complicaciones, y puede ser útil en el seguimiento de los pacientes de alto riesgo.

Los hallazgos de la radiografía de tórax son inespecíficos y pueden ser ocasionados por múltiples enfermedades infecciosas y no infecciosas que afectan el parénquima pulmonar. De este modo, el diagnóstico de neumonía debe estar sustentado en un cuadro clínico compatible (fiebre, tos, expectoración, calofríos, dolor torácico, disnea) asociado a la presencia de infiltrados pulmonares de aparición reciente en la radiografía de tórax. La resolución de los infiltrados radiográficos a menudo ocurre varias semanas o meses después de la mejoría clínica, especialmente en el adulto mayor, pacientes con neumonía multilobar o bilateral, neumonía bacteriémica y NAC grave manejada en la $\mathrm{UCI}^{14}$.

\section{Diagnóstico Microbiológico Y EXÁMENES COMPLEMENTARIOS}

Patricio Jiménez $\mathrm{P}^{1}$, Mario Calvo $\mathrm{A}^{2}$ y Comité de Microbiología de la Sociedad Chilena de Infectología El estudio microbiológico en pacientes con neumonía comunitaria permite identificar el agente causal de la neumonía, su patrón de sensibilidad a antimicrobianos y orientar el tratamiento antibiótico específico para cada paciente. El tratamiento antimicrobiano dirigido permite reducir su espectro de acción, disminuyendo los costos, el riesgo de reacciones adversas y el desarrollo de cepas multirresistentes. Sin embargo, la sensibilidad y especificidad de los exámenes microbiológicos son sólo moderados y, a menudo, no contribuyen al manejo inicial del paciente con NAC ${ }^{15,16}$. Así, en un estudio nacional, que incluyó 463 adultos inmunocompetentes con NAC, ingresados en un hospital docente de la Región Metropolitana, sólo se pudo determinar la etiología en $25 \%$ de los casos $^{17}$

El estudio etiológico por ningún motivo debe retrasar el inicio del tratamiento antibiótico y los cuidados generales del enfermo, ya que este retraso puede incrementar la mortalidad por neumonía ${ }^{18}$. De este modo, no es necesario realizar estudios microbiológicos extensos en todos los pacientes con neumonía comunitaria. Los estudios deben estar guiados por la gravedad de la neumonía, los factores de riesgo epidemiológico y la respuesta al tratamiento empírico. No se recomienda realizar exámenes microbiológicos de rutina en los pacientes sin criterios de gravedad manejados en el medio ambulatorio. Sin embargo, en los pacientes con tos productiva, expectoración persistente y compromiso del estado general, se recomienda obtener muestras de expectoración para baciloscopias y cultivo de Koch.

La gravedad y riesgo de muerte (10-30\%) de los enfermos hospitalizados por neumonía comunitaria, justifica la realización de exámenes microbiológicos básicos (tinción de Gram y cultivo de expectoración, hemocultivos, cultivo de líquido pleural), intentando pesquisar el agente causal de la infección pulmonar y orientar el tratamiento antimicrobiano específico ${ }^{8,12,17}$.

Además, se recomienda obtener muestras de suero pareadas para la medición de títulos de anticuerpos de patógenos atípicos (M pneumoniae, C pneumoniae) y muestra de orina para la detección del antígeno urinario de Legionella pneumophila en todos los pacientes que no responden al tratamiento empírico con agentes ß-lactámicos, en los pacientes con NAC grave admitidos a la UCI y en casos seleccionados con factores de riesgo epidemiológico específicos. La detección de antígenos de virus influenza A y $B$, se recomienda en adultos con neumonía que requieren ser hospitalizados y en ancianos durante la época de alta circulación de virus respiratorios en la comunidad.

Los exámenes de laboratorio hematológicos y bioquímicos no son útiles para confirmar el diagnóstico ni la etiología de la neumonía. Sin embargo, pueden ser empleados para evaluar la gravedad del episodio, establecer el pronóstico y orientar el lugar de manejo (ambulatorio o admisión al hospital). En general, en los pacientes con neumonía comunitaria sin comorbilidad o factores de riesgo de manejo ambulatorio, no es necesario solicitar exámenes de laboratorio complementarios. En los pacientes con NAC y patologías concomitantes, es preferible obtener muestras para hemograma, pruebas de función renal y hepática, glicemia y proteína $\mathrm{C}$ reactiva. La medición de la saturación de hemoglobina mediante oximetría de pulso puede ser útil en estos casos. En los pacientes que ingresan al hospital es recomendable, además de los exámenes descritos anteriormente, medir gases en sangre arterial por su valor pronóstico y terapéutico. 


\section{ETIOLOGÍA}

Rodrigo Moreno $\mathrm{B}^{1}$, Raúl Riquelme $\mathrm{O}^{1}$.

En la situación clínica ideal, el tratamiento antimicrobiano empírico prescrito en la NAC debería estar basado en el resultado de los estudios microbiológicos efectuados en distintas áreas geográficas del medio nacional. Sin embargo, la información disponible sobre la etiología en el ámbito ambulatorio y la UCI es relativamente escasa, en comparación con el medio intrahospitalario.

En los estudios diseñados específicamente para buscar agentes causales, en el 40-50\% de los casos no se identifica el patógeno respiratorio, lo que pone de manifiesto las dificultades de los métodos diagnósticos ${ }^{19}$. En todos los entornos de atención, el Streptococcus pneumoniae es el principal patógeno respiratorio aislado en la neumonía comunitaria del adulto, siendo responsable de $16 \%$ de los casos tratados en el medio ambulatorio y de alrededor del $22 \%$ de los casos admitidos al hospital y la UCI ${ }^{4,5,12}$. Se estima que un tercio de los casos son ocasionados por un conjunto de microorganismos: Haemophilus influenzae, Mycoplasma pneumoniae, Chlamydia pneumoniae, virus respiratorios (influenza, parainfluenza, sincicial respiratorio y adenovirus), $\mathrm{S}$ aureus, bacilos Gram negativos entéricos, Legionella sp y anaerobios estrictos, cada uno de los cuales es responsable, en general, de menos de $10 \%$ de los casos. La distribución de los microorganismos varía escasamente en los tres entornos de atención: ambulatorio, sala de cuidados generales y UCI. Entre las excepciones destaca una mayor frecuencia de infección por bacilos Gram negativos, S aureus y Legionella sp en la UCI y de Chlamydia pneumoniae en el medio ambulatorio.

En Chile, la etiología de la NAC en pacientes hospitalizados es similar a la comunicada en estudios extranjeros ${ }^{20-23}$. Aún no se dispone de información sobre la etiología de la NAC manejada en el medio ambulatorio y la NAC grave manejada en la UCI. En los estudios diseñados específicamente para estudiar los agentes causales de la neumonía en el medio nacional, en 50-75\% de los casos no se logró identificar el agente patógeno (Tabla 1). En el ambiente hospitalario, el

Tabla 1. Etiología de la neumonía adquirida en la comunidad en el adulto hospitalizado en Chile

\begin{tabular}{|lcccc|}
\hline Etiología de la NAC & Trucco $^{20}$ & Saldías $^{21}$ & Riquelme $^{22}$ & Díaz $^{23}$ \\
\hline $\mathrm{N}^{\circ}$ pacientes & 140 & 463 & 200 & 130 \\
Duración del estudio (meses) & 21 & 24 & 12 & 16 \\
Entorno del estudio & Hospital & Hospital & Hospital & Hospital \\
Edad promedio (años) & 60 & 66 & 63 & 68 \\
Comorbilidad (\%) & 51 & 67 & 75 & 71 \\
Patógenos respiratorios (\%) & & & & \\
S pneumoniae & 5,7 & 10,2 & 12 & 16,9 \\
H influenzae & 2,8 & 3,7 & 7 & 2,3 \\
Gram negativos & 7,8 & 5,2 & 4 & 3,1 \\
S aureus & 5,7 & 2,8 & 3,5 & 0,7 \\
M pneumoniae & - & $4 / 42$ & 0,5 & 3,1 \\
C pneumoniae & - & - & 5 & 3,1 \\
Legionella sp & 8,5 & $1 / 36$ & 1,5 & 1,5 \\
Influenza A y B & - & $6 / 28$ & - & 6,9 \\
Otros virus & - & - & - & 12,4 \\
Mixta & - & - & 5 & - \\
Otros & 2,8 & - & - & 51 \\
Desconocida & 76 & 76 & 70,5 & \\
\hline
\end{tabular}


Streptococcus pneumoniae es el principal patógeno respiratorio aislado, y le siguen en importancia un conjunto de varios microorganismos: $\mathrm{H}$ influenzae, bacilos Gram negativos, S aureus, M pneumoniae, C pneumoniae, Legionella sp y los virus respiratorios. Considerando las escasas y previsibles diferencias entre los microorganismos causales de las neumonías manejadas en diferentes ámbitos de atención, es razonable proponer que mientras no se disponga de resultados nacionales en el medio extrahospitalario y la UCI, se continúen haciendo extensivos a nuestro país los resultados de estudios efectuados en otras áreas geográficas.

\section{EVALUACIÓN DE LA GRAVEDAD}

Fernando Saldías $\mathrm{P}^{1}$, Juana Pavié $\mathrm{G}^{1}$.

El cuadro clínico del paciente con NAC que solicita atención en el medio ambulatorio (consultorios y Servicios de Urgencia), puede variar entre un cuadro infeccioso leve de bajo riesgo de complicaciones hasta uno de extrema gravedad con riesgo vital 8,12,17. El riesgo de complicaciones y muerte del paciente con neumonía comunitaria que no tiene criterios de gravedad, manejado en el ámbito ambulatorio (letalidad inferior a 1-2\%) es bastante bajo comparado con los pacientes admitidos al hospital (letalidad: 10-20\%); siendo especialmente elevado en aquellos con criterios de neumonía grave admitidos a las unidades de cuidado crítico (letalidad: 20-50\%)8,17,21. Se ha observado una gran variabilidad en la tasa de hospitalizaciones por neumonía en diferentes áreas geográficas, probablemente determinado por diferentes criterios empleados por los médicos para evaluar la gravedad de los enfermos, accesibilidad a los sistemas de salud y las características de la población examinada.

Una vez establecido el diagnóstico clínicoradiográfico de neumonía, el principal objetivo del médico será evaluar la gravedad del enfermo, lo cual le permitirá predecir su pronóstico, decidir el lugar de manejo (ambulatorio, sala 0 UCI), la extensión del estudio microbiológico y exámenes complementarios, y orientar el tratamiento antimicrobiano empírico (fármacos, ruta, duración).
En la Tabla 2 se describen los principales factores pronósticos asociados a evolución clínica desfavorable y riesgo de muerte elevado en adultos hospitalizados con neumonía adquirida en la comunidad ${ }^{4-7}$.

Recomendaciones para la evaluación de la gravedad en los pacientes con neumonía comunitaria. Se recomienda a los clínicos implementar una estrategia simple y práctica para evaluar la gravedad y riesgo de complicaciones de los pacientes con NAC atendidos en los servicios de atención primaria.

En el ámbito ambulatorio, donde no se dispone de exámenes complementarios, se recomienda evaluar la gravedad de los pacientes con NAC considerando las variables de la Figura 1.

En ausencia de factores de riesgo se recomienda manejo ambulatorio, en presencia de un factor de riesgo se recomienda manejo ambulatorio o en el hospital según la experiencia previa y el juicio clínico, en presencia de dos o más factores de riesgo se recomienda referir al hospital. El juicio clínico y la experiencia del médico deben predominar sobre los modelos predictores, los cuales no son infalibles. El médico debería siempre considerar las preferencias y requerimientos de los enfermos en la toma de decisiones acerca del lugar de manejo y tratamiento prescrito.

\section{NeUMONÍA COMUNITARIA GRAVE}

Francisco Arancibia $\mathrm{H}^{1}$, Orlando Díaz $\mathrm{P}^{1}$.

Las neumonías adquiridas en la comunidad que requieren tratamiento en la Unidad de Cuidados Intensivos representan entre 10 y $30 \%$ de los pacientes hospitalizados por $\mathrm{NAC}^{4,8,21}$. En los pacientes con NAC grave la tasa de complicaciones, estadía en el hospital y mortalidad son elevadas, variando esta última entre $21 \%$ y $54 \%$, según distintas series ${ }^{4,5,8}$.

Definición de NAC grave. El paciente con neumonía comunitaria grave es aquel que necesita vigilancia y monitorización de una unidad de cuidados intensivos, donde, si es necesario, puede recibir apoyo especializado con conexión a un ventilador mecánico, soporte hemodinámico, o ambos (Tabla 3). 
Tabla 2. Factores pronósticos asociados a evolución clínica desfavorable y/o riesgo de muerte en adultos con neumonía adquirida en la comunidad

1. Variables sociodemográficas

- Edad avanzada (mayores de 65 años).

- Lugar de procedencia (centro geriátrico).

2. Cuadro clínico

Historia:

- Disnea

- Compromiso de conciencia

- Sospecha de aspiración (trastornos de la deglución y/o compromiso del sensorio)

- Comorbilidades específicas: cardiopatías, enfermedad pulmonar crónica (EPOC, bronquiectasias), diabetes mellitus, enfermedad cerebrovascular con secuela motora o deterioro psicoorgánico severo, insuficiencia renal crónica, alcoholismo, malnutrición, enfermedad hepática crónica, cáncer.

Examen físico:

- Frecuencia cardíaca >120 latidos/min

- Presión arterial $<90 / 60 \mathrm{mmHg}$

- Frecuencia respiratoria $\geq 20 \mathrm{resp} / \mathrm{min}$

- Ausencia de fiebre $\left(<37^{\circ} \mathrm{C}\right)$ o hipertermia $\left(>40^{\circ} \mathrm{C}\right)$

- Compromiso de conciencia: somnolencia, sopor, coma, confusión mental.

3. Radiografía de tórax:

- Compromiso radiográfico multilobar o bilateral

- Derrame pleural

- Cavitación o absceso pulmonar

4. Exámenes de laboratorio:

- Función renal anormal: nitrógeno ureico $>20 \mathrm{mg} / \mathrm{dl}$ o creatininemia $>1,2 \mathrm{mg} / \mathrm{dl}$

- Hipoxemia: $\mathrm{PaO}_{2}<60 \mathrm{mmHg}$ respirando aire ambiente

- Hipercapnia: $\mathrm{PaCO}_{2}>50 \mathrm{mmHg}$ respirando aire ambiente

- Anemia: hematocrito $<30 \%$ o hemoglobina $<9 \mathrm{~g} / \mathrm{dl}$

- Leucocitosis $>30.000$ células $/ \mathrm{mm}^{3}$ o leucopenia $<4.000$ células $/ \mathrm{mm}^{3}$

5. Exámenes microbiológicos:

- Neumonía bacteriémica con hemocultivos positivos

- Infección pulmonar por bacilos Gram negativos entéricos, Saureus, K pneumoniae, P aeruginosa y Legionella sp.

En todos los pacientes con neumonía comunitaria se recomienda evaluar la gravedad de la infección en el momento de su admisión al hospital. Esta evaluación es preferible realizarla junto a un médico con experiencia, y si presenta criterios de mal pronóstico se sugiere trasladar precozmente a la UCI. Son útiles para esta evaluación los criterios de la ATS y los criterios de la BTS modificados (CURB) $)^{4,5}$. El juicio clínico y la experiencia del médico deben predominar sobre los modelos predictores, los cuales no son infalibles.
Exámenes microbiológicos. En los pacientes con neumonía comunitaria grave se recomienda solicitar los siguientes exámenes microbiológicos: tinción de Gram y cultivo de expectoración, hemocultivos aerobios, Gram y cultivo de líquido pleural si hay derrame pleural puncionable, antígeno urinario de S pneumoniae y Legionella pneumophila, hisopado nasofaríngeo para la búsqueda de antígenos de virus influenza A y B (durante el período epidémico de otoño-invierno), y serología para microorganismos atípicos ( $\mathrm{M}$ pneumoniae y $\mathrm{C}$ pneumoniae). 


\section{Recomendaciones para la evaluación de la gravedad del paciente CON NEUMONÍA COMUNITARIA ATENDIDO EN EL ÁMBITO AMBULATORIO}

- $\quad$ Edad mayor de 65 años.

- Presencia de comorbilidad: Cardiopatía coronaria, insuficiencia cardíaca, EPOC, bronquiectasias, diabetes, enfermedad cerebrovascular con trastorno de deglución, insuficiencia renal crónica, enfermedad hepática crónica, alcoholismo, neoplasia activa

- Estado mental alterado: somnolencia, sopor, coma o confusión mental.

- Frecuencia cardíaca $\geq 120$ latidos/min.

- Hipotensión arterial (PA $<90 / 60 \mathrm{mmHg}$ ).

- Frecuencia respiratoria $\geq 20 \mathrm{resp} / \mathrm{min}$.

- Rx tórax: NAC multilobar, cavitación, derrame pleural.

- $\quad \mathrm{SaO}_{2}<90 \%$ con $\mathrm{FiO}_{2}$ ambiental.

- Presencia de comorbilidad descompensada.

- Factores sociales desfavorables y problemas de adherencia al tratamiento.

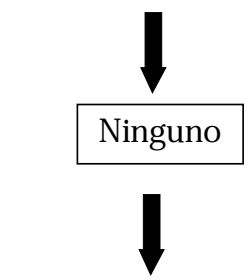

Manejo ambulatorio
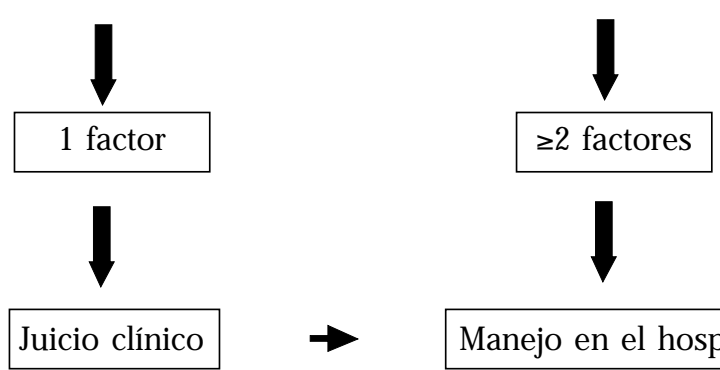

Figura 1.

\section{Tabla 3. C riterios de neumonía comunitaria grave según la Sociedad Americana de T órax y la Sociedad Británica de T órax}

Sociedad Americana de Tórax ${ }^{4}$ (ATS):

Criterios mayores (presencia de 1 criterio)

* Necesidad de ventilación mecánica

* Presencia de shock séptico

Criterios menores (presencia de $\geq 2$ criterios)

* Presión arterial sistólica $<90 \mathrm{mmHg}$

* Compromiso radiográfico multilobar

* $\mathrm{PaO}_{2} / \mathrm{FiO}_{2}<250 \mathrm{mmHg}$

Sociedad Británica de Tórax ${ }^{5}$ (BTS) ( $\geq 2$ criterios)

* Frecuencia respiratoria mayor de $30 \mathrm{resp} /$ $\min$

* Presión diastólica menor de $60 \mathrm{mmHg}$

* Nitrógeno ureico $>20 \mathrm{mg} / \mathrm{dl}$

* Confusión mental de reciente aparición
8. TRATAMiento

Alejandro Díaz $\mathrm{F}^{1}$, Jaime Labarca L2 ${ }^{2}$, Carlos Pérez $\mathrm{C}^{2}$, Mauricio Ruiz $\mathrm{C}^{1}$, Marcelo Wolff $\mathrm{R}^{2}$.

El tratamiento antibiótico apropiado reduce la duración de la sintomatología asociada a la neumonía, el riesgo de complicaciones y la mortalidad. En la mayoría de los casos, no es posible identificar el agente microbiológico que ocasiona la infección y por esto el tratamiento antibiótico se prescribe en forma empírica ${ }^{4-7,20-23}$. Los antecedentes epidemiológicos, la presencia de comorbilidad y la estimación de la gravedad son los principales factores que determinan el pronóstico del paciente con neumonía comunitaria y por lo tanto deben ser considerados para decidir el lugar de manejo y la elección del tratamiento antimicrobiano empírico.

Resistencia de Streptococcus pneumoniae a los antibióticos. En Chile, un tercio de las cepas de S 
pneumoniae muestra susceptibilidad disminuida a penicilina y en $18 \%$ de los casos la resistencia es alta $(\mathrm{CIM} \geq 2 \mu \mathrm{g} / \mathrm{ml})$; mientras que la resistencia a enitromicina fluctúa entre $10-15 \%$ y a cefotaxima entre 2-10\%24,25. La implicancia clínica de la resistencia a antibióticos de Spneumoniae es controvertida, ya que algunos estudios demostraron que la resistencia a penicilina con niveles de CIM entre 0,1 y 2 $\mu \mathrm{g} / \mathrm{ml}$ no implicaron mayor riesgo de complicaciones y muerte. Sin embargo, se ha comunicado un aumento de la letalidad en pacientes con neumonía neumocócica con CIM para penicilina $\geq 4 \mu \mathrm{g} / \mathrm{ml}^{26}$. Afortunadamente, no se han comunicado cepas con este nivel de resistencia en la población adulta de nuestro país. Varios estudios han demostrado la eficacia de los agentes ß-lactámicos en el tratamiento de la neumonía neumocócica con CIM para penicilina inferior a $4 \mu \mathrm{g} / \mathrm{ml}^{27}$.

La emergencia de cepas de S pneumoniae resistentes a antimicrobianos en nuestro medio es una realidad. Se ha asociado al uso indiscriminado de antibióticos en la patología respiratoria de origen viral, y consideramos que es responsabilidad de los médicos educar a la población sobre este tema y prescribir los antimicrobianos racionalmente, para evitar el incremento sostenido de este problema en nuestro medio.
Los pacientes con neumonía comunitaria se han agrupado en cuatro categorías de riesgo:

Grupo 1: Pacientes menores de 65 años sin comorbilidad.

Grupo 2: Pacientes mayores de 65 años o con comorbilidad de manejo ambulatorio.

Grupo 3: Pacientes hospitalizados en sala de cuidados generales que tienen criterios de gravedad moderada.

Grupo 4: Pacientes con NAC grave que deben ser manejados en la Unidad de Cuidados Intermedios o Intensivos.

Los pacientes sin criterios de gravedad seleccionados apropiadamente para manejo ambulatorio tienen bajo riesgo de complicaciones y la letalidad es inferior a 1-2\%. Los agentes etiológicos más frecuentes en esta categoría son $\mathrm{S}$ pneumoniae, H influenzae, Mycoplasma pneumoniae, Chlamydia pneumoniae y los virus respiratorios $^{4-6}$. El tratamiento antibiótico empírico de la neumonía ambulatoria debe cubrir fundamentalmente a $\mathrm{S}$ pneumoniae y $\mathrm{H}$ influenzae, y ocasionalmente es necesario cubrir los llamados agentes atípicos (Mycoplasma, Chlamydia y Legionella).

\section{Tabla 4. Recomendaciones de tratamiento antimicrobiano empírico para pacientes con neumonía comunitaria manejados en el ámbito ambulatorio}

\begin{tabular}{|c|c|c|}
\hline Categoría de la neumonía & $\begin{array}{l}\text { Antibiótico de elección, dosis, vía y } \\
\text { duración del tratamiento }\end{array}$ & Régimen alternativo \\
\hline $\begin{array}{l}\text { Grupo 1: menores de } 65 \\
\text { años sin comorbilidad y } \\
\text { factores de riesgo }\end{array}$ & $\begin{array}{l}\text { Amoxicilina } 1 \text { gramo cada } 8 \mathrm{~h} \\
\text { vía oral por } 7 \text { días }\end{array}$ & $\begin{array}{l}\text { Eritromicina } 500 \mathrm{mg} \text { cada } 6 \mathrm{~h} \\
\text { vía oral por } 7 \text { días } \\
\text { Claritromicina } 500 \mathrm{mg} \text { cada } 12 \mathrm{~h} \\
\text { vía oral por } 7 \text { días, o } \\
\text { Azitromicina } 500 \mathrm{mg} / \text { día vía oral } \\
\text { por } 5 \text { días }\end{array}$ \\
\hline $\begin{array}{l}\text { Grupo 2: mayores de } 65 \\
\text { años o de cualquier edad con } \\
\text { comorbilidad específica }\end{array}$ & $\begin{array}{l}\text { Amoxicilina-ácido clavulánico } \\
500 / 125 \text { mg cada } 8 \text { h } 0 \text { 875/125 } \\
\text { mg cada } 12 \text { h vía oral por } 7 \text { días, } \\
\text { o } \\
\text { Cefuroxima } 500 \text { mg cada } 12 \text { h } \\
\text { vía oral por } 7 \text { días }\end{array}$ & $\begin{array}{l}\text { Eritromicina } 500 \mathrm{mg} \text { cada } 6 \mathrm{~h} \\
\text { vía oral por } 7 \text { días, } \\
\text { Claritromicina } 500 \mathrm{mg} \text { cada } 12 \mathrm{~h} \\
\text { vía oral por } 7 \text { días, o } \\
\text { Azitromicina } 500 \mathrm{mg} / \text { día vía oral } \\
\text { por } 5 \text { días }\end{array}$ \\
\hline
\end{tabular}


Recomendaciones para pacientes con NAC de bajo riesgo, de manejo ambulatorio.

1. Ante la sospecha clínica de una neumonía se sugiere confirmar el diagnóstico con una radiografía de tórax; si no es posible obtenerla, se sugiere tratar al paciente como si tuviera una infección pulmonar, porque el pronóstico del paciente empeora cuando se retrasa el inicio del tratamiento antibiótico ${ }^{18}$.

2. Es importante aplicar una evaluación objetiva de la gravedad y determinar si existe algún criterio de hospitalización ${ }^{8,12,17}$. El manejo ambulatorio de un paciente con neumonía implica la ausencia de criterios clínicos y/o sociales de riesgo que recomienden su hospitalización.

3. Cuando sea factible, la evaluación del paciente debe considerar la medición de la saturación arterial de oxígeno mediante oximetría de pulso, y si la $\mathrm{SaO}_{2}$ es inferior a $90 \%$, se recomienda derivar el enfermo al hospital para corregir la insuficiencia respiratoria.

4. El antibiótico prescrito debe ser administrado precozmente, idealmente dentro de las $8 \mathrm{~h}$ de realizado el diagnóstico.
5. El paciente debe ser enviado a su domicilio con indicación de reposo, control de temperatura, hidratación oral e inicio del tratamiento antimicrobiano vía oral.

6. El paciente debe acudir a control al finalizar el tratamiento antimicrobiano o inmediatamente en caso de evolución desfavorable: persistencia de la fiebre por más de tres días, aumento de la dificultad respiratoria o compromiso del estado general, aparición de criterios de gravedad.

7. El tratamiento antimicrobiano debe durar siete días, excepto cuando se indica azitromicina con la que bastarían cinco días.

Los pacientes con neumonía comunitaria, admitidos a la sala de cuidados generales, tienen un riesgo intermedio de complicaciones y muerte comparados con aquellos tratados en el medio ambulatorio y los internados en unidades de cuidado crítico $^{8,21}$. En Chile, la letalidad de los pacientes con neumonía hospitalizados en sala de cuidados generales varía entre 3 y $20 \%^{20-23}$. Los patógenos respiratorios más relevantes en esta categoría son el S pneumoniae, $\mathrm{H}$ influenzae, los

\section{Tabla 5. Recomendaciones de tratamiento antimicrobiano empírico para pacientes con neumonía comunitaria de gravedad moderada manejados en sala de cuidados generales}

\begin{tabular}{|c|c|c|}
\hline Categoría de la neumonía & $\begin{array}{l}\text { Antibiótico de elección, dosis, vía y } \\
\text { duración del tratamiento }\end{array}$ & Régimen alternativo \\
\hline $\begin{array}{l}\text { Grupo 3: pacientes de } \\
\text { cualquier edad, con } \\
\text { criterios de gravedad } \\
\text { moderada, hospitalizados } \\
\text { en sala de cuidados } \\
\text { generales }\end{array}$ & $\begin{array}{l}\text { Ceftriaxona } 1-2 \mathrm{~g} / \text { día iv, } 0 \\
\text { Cefotaxima } 1 \mathrm{~g} \text { cada } 8 \mathrm{~h} \text { iv } \\
\text { Duración: } 7-10 \text { días } \\
\text { En presencia de: } \\
\text { - Fracaso de tratamiento con agentes ß- } \\
\text { lactámicos } \\
\text {-Serología positiva para Mycoplasma, } \\
\text { Chlamydia o Legionella sp } \\
\text { Se recomienda agregar: } \\
\text { Eritromicina } 500 \mathrm{mg} \text { cada } 6 \mathrm{~h} \text { iv u oral, } \\
\text { Claritromicina } 500 \mathrm{mg} \text { cada } 12 \mathrm{~h} \text { oral, } 0 \\
\text { Azitromicina } 500 \mathrm{mg} / \text { día oral }\end{array}$ & $\begin{array}{l}\text { Amoxicilina-ácido clavulánico } \\
1.000 / 200 \mathrm{mg} \text { c/ } 8 \mathrm{~h} \text { iv, } \\
\text { Amoxicilina-sulbactam } \\
1.000 / 500 \mathrm{mg} \text { cada } 8 \mathrm{~h} \text { iv, } 0 \\
\text { Ampicilina-sulbactam } \\
1.000 / 500 \mathrm{mg} \text { cada } 8 \mathrm{~h} \text { iv } \\
\text { En presencia de: } \\
\text { - Fracaso de tratamiento con } \\
\text { agentes ß-lactámicos. } \\
\text { Serología positiva para Mycoplasma, } \\
\text { Chlamydia o Legionella sp } \\
\text { Se recomienda agregar: } \\
\text { Eritromicina } 500 \mathrm{mg} \text { cada } 6 \mathrm{~h} \text { iv u oral, } \\
\text { Claritromicina } 500 \mathrm{mg} \text { cada } 12 \mathrm{~h} \text { oral, } 0 \\
\text { Azitromicina } 500 \mathrm{mg} / \text { día oral }\end{array}$ \\
\hline
\end{tabular}


gérmenes atípicos ( $\mathrm{M}$ pneumoniae, $\mathrm{C}$ pneumoniae y Legionella sp), los bacilos Gram negativos entéricos y los anaerobios ${ }^{4,5}$. Los estudios clínicos aún no han esclarecido cuándo es conveniente que los pacientes, con neumonía comunitaria de gravedad intermedia hospitalizados en sala, deban ser tratados con un agente ß-lactámico o la combinación de ßs-lactámico y un macrólido 28 .

Recomendaciones para pacientes con NAC de riesgo moderado hospitalizados en sala.

1. En el Servicio de Urgencia se debe evaluar la gravedad del enfermo y medir la saturación arterial de oxígeno con oximetría de pulso: si la $\mathrm{SaO}_{2}$ es inferior a $90 \%$ se debe corregir la insuficiencia respiratoria, administrando oxígeno por naricera o mascarilla y se debe medir gases arteriales.

2. Solicitar dos hemocultivos aeróbicos antes de iniciar el tratamiento antimicrobiano.

3. El antibiótico prescrito debe ser administrado precozmente, idealmente dentro de las primeras ocho horas de realizado el diagnóstico.

4. Realizar la técnica de pesquisa rápida de virus respiratorios de hisopado o aspirado nasofaríngeo durante la época de alta circulación de virus en la comunidad.

5. Si se detecta infección por virus influenza A o $B$ se debe indicar aislamiento respiratorio en cohorte y prescribir terapia antiviral.
6. La duración del tratamiento antimicrobiano en la neumonía comunitaria de gravedad moderada que requiere hospitalización es 7 a 10 días.

En la categoría de NAC grave se concentran los pacientes con alto riesgo de complicaciones y muerte ${ }^{8,21}$. La letalidad de la neumonía comunitaria grave manejada en la UCI fluctúa entre 17 y 50\%. Los patógenos más importantes aislados en pacientes con NAC grave son S pneumoniae, bacilos Gram negativos entéricos, Legionella sp, S aureus, $\mathrm{H}$ influenzae, anaerobios estrictos, Chlamydia pneumoniae, Mycoplasma pneumoniae y los virus respiratorios $4,5,21$. Debido al elevado riesgo de complicaciones y muerte asociados a la NAC grave, se recomienda la administración de tratamiento antibiótico combinado vía endovenosa con prontitud, una vez establecido el diagnóstico.

Recomendaciones para pacientes hospitalizados con neumonía comunitaria grave.

1. El tratamiento antibiótico empírico deber ser combinado (ß-lactámico asociado a macrólidos o fluoroquinolonas) y administrado por vía parenteral.

2. El antibiótico prescrito debe ser administrado precozmente, idealmente dentro de las primeras cuatro horas de realizado el diagnóstico.

3. En general, la duración del tratamiento antimicrobiano fluctúa entre 10 y 14 días, dependiendo de la evolución clínica y el agente causal de la neumonía.

Tabla 6. Recomendaciones de tratamiento antimicrobiano empírico en la neumonía comunitaria grave

\begin{tabular}{|c|c|c|}
\hline $\begin{array}{l}\text { Categoría de la } \\
\text { Neumonía }\end{array}$ & $\begin{array}{l}\text { Antibiótico de elección, dosis, vía y } \\
\text { duración del tratamiento }\end{array}$ & Régimen alternativo \\
\hline $\begin{array}{l}\text { Grupo 4: pacientes con } \\
\text { neumonía comunitaria } \\
\text { grave hospitalizados en la } \\
\text { Unidad de Intermedio o UCI }\end{array}$ & $\begin{array}{l}\text { Ceftriaxona } 2 \mathrm{~g} / \text { día iv, } 0 \\
\text { Cefotaxima } 1-2 \mathrm{~g} \text { cada } 8 \mathrm{~h} \text { iv } \\
\text { Asociado a: } \\
\text { Eritromicina } 500 \mathrm{mg} \text { cada } 6 \mathrm{~h} \text { iv, } \\
\text { Levofloxacina } 500-1.000 \mathrm{mg} / \text { día iv, } 0 \\
\text { Moxifloxacina } 400 \mathrm{mg} / \text { día iv } \\
\text { Duración: } 10-14 \text { días }\end{array}$ & $\begin{array}{l}\text { Amoxicilina-ácido clavulánico 1.000/200 } \\
\text { mg cada } 8 \mathrm{~h} \text { iv, } 0 \\
\text { Amoxicilina-sulbactam 1.000/500 mg cada } \\
8 \mathrm{~h} \text { iv, } 0 \\
\text { Ampicilina-sulbactam 1.000/500 mg cada } \\
8 \mathrm{~h} \text { iv } \\
\text { Asociado a: } \\
\text { Eritromicina } 500 \mathrm{mg} \text { cada } 6 \mathrm{~h} \text { iv, } \\
\text { Levofloxacina } 500-1000 \mathrm{mg} / \text { día iv, } 0 \\
\text { Moxifloxacina } 400 \mathrm{mg} / \text { día iv }\end{array}$ \\
\hline
\end{tabular}


Tratamiento antimicrobiano específico según agente causal de la neumonía comunitaria.

- Streptococcus pneumoniae: Amoxicilina 750$1.000 \mathrm{mg}$ cada $8 \mathrm{~h}$ oral, Eritromicina $500 \mathrm{mg}$ cada $6 \mathrm{~h}$ oral o iv, Claritromicina $500 \mathrm{mg}$ cada $12 \mathrm{~h}$ oral, Cefuroxima $500 \mathrm{mg}$ cada $12 \mathrm{~h}$ oral o 750 mg cada 8 h iv, Ceftriaxona 1-2 g/ día iv 0 Cefotaxima $1 \mathrm{~g}$ cada $8 \mathrm{~h}$ iv.

Duración del tratamiento: 7-10 días.

- Mycoplasma pneumoniae y Chlamydia pneumoniae: Eritromicina $500 \mathrm{mg}$ cada $6 \mathrm{~h}$ oral 0 iv, Claritromicina $500 \mathrm{mg}$ cada $12 \mathrm{~h}$ oral, Tetraciclina 500 mg cada 6 h oral, Azitromicina $500 \mathrm{mg} /$ día oral.

Duración del tratamiento: 14 días (excepto con Azitromicina que se recomiendan 5 días).

- Legionella sp: Claritromicina $500 \mathrm{mg}$ cada $12 \mathrm{~h}$ oral, Levofloxacina $500 \mathrm{mg}$ cada $12 \mathrm{~h}$ oral 0 $1.000 \mathrm{mg} /$ día iv, Moxifloxacina $400 \mathrm{mg} /$ día oral o iv o Gatifloxacina $400 \mathrm{mg} /$ día oral, asociado a Rifampicina $600 \mathrm{mg}$ cada $12 \mathrm{~h}$ oral. Duración del tratamiento: 14-21 días.

- Haemophilus influenzae no productor de betalactamasa: Amoxicilina 750-1.000 mg cada 8 h oral.

- Haemophilus influenzae productor de betalactamasa: Amoxicilina-ácido clavulánico 500/125 mg cada 8 h o 875/125 mg cada $12 \mathrm{~h}$ oral, Cefuroxima $750 \mathrm{mg}$ cada $8 \mathrm{~h}$ iv o $500 \mathrm{mg}$ cada $12 \mathrm{~h}$ oral, Ceftriaxona 1-2 g/día iv o Cefotaxima $1 \mathrm{~g}$ cada $8 \mathrm{~h}$ iv.

Duración del tratamiento: 10-14 días.

- Bacilos Gram negativos entéricos: Cefotaxima 1-2 g cada $8 \mathrm{~h}$ iv o Ceftriaxona 1-2 g/día iv. Duración del tratamiento: 14-21 días.

- Pseudomonas aeruginosa: Ceftazidima $2 \mathrm{~g}$ cada 8 h iv o Piperacilina/Tazobactam $4,5 \mathrm{~g}$ cada 8 h iv, asociado a Ciprofloxacina 500-750 mg cada $12 \mathrm{~h}$ oral 0 iv.

Duración del tratamiento: 14-21 días.

- Staphylococcus aureus meticilina sensible: Cloxacilina 500-1.000 mg cada $6 \mathrm{~h}$ oral o iv.

- Staphylococcus aureus meticilina resistente: Vancomicina $1 \mathrm{~g}$ cada $12 \mathrm{~h}$ iv.

Duración del tratamiento: 14-21 días.

En la práctica clínica, sólo en 20 a 30\% de los casos de neumonía comunitaria que requieren hospitalización se logra identificar el agente causal ${ }^{12,20-23}$. De acuerdo al patógeno respiratorio identificado se recomienda el tratamiento antimicrobiano específico. Sin embargo, la elección del esquema antibiótico y la vía de administración deben basarse no sólo en la información microbiológica, sino además debe considerar el resultado del antibiograma, la condición general y gravedad del paciente y la posibilidad de una infección polimicrobiana.

Criterios de estabilidad clínica, cambio a tratamiento antibiótico oral y egreso hospitalario. La mayoría de los pacientes con NAC alcanzan la estabilidad clínica entre el tercer y quinto día en el hospital. La estabilidad clínica se produce cuando se normalizan los signos vitales, el estado mental es normal o retorna a la condición basal y mejora el intercambio gaseoso disminuyendo los requerimientos de oxígeno ${ }^{29}$. La aplicación correcta de los criterios de estabilidad clínica para realizar el cambio del tratamiento antibiótico a la vía oral permite disminuir la duración de la hospitalización sin aumentar los riesgos para el enfermo.

Criterios de estabilidad clínica para decidir el cambio del antibiótico a la vía oral:

1. Signos vitales estables durante $24 \mathrm{~h}$ : frecuencia cardíaca $\leq 100$ lat/min, presión arterial sistólica $\geq 90 \mathrm{mmHg}$, frecuencia respiratoria $\leq 24 \mathrm{resp} /$ min, temperatura $<37,8^{\circ} \mathrm{C}$.

2. Mejoría significativa $\mathrm{y} / 0$ resolución de los síntomas respiratorios.

3. Ausencia o disminución de los requerimientos de oxígeno.

4. Estado mental normal o retorno a la condición basal.

5. Tubo digestivo funcionante o capaz de ingerir el antibiótico vía oral.

Criterios de estabilidad clínica para decidir el alta hospitalaria:

1. Haber cumplido los criterios de cambio a la vía oral.

2. $\mathrm{SaO}_{2} \geq 90 \%$ respirando aire ambiente o con bajo flujo de oxígeno.

3. Las comorbilidades deben estar compensadas.

4. Ausencia de factores psicosociales que contraindiquen el manejo ambulatorio.

Antibióticos prescritos en el cambio a la vía oral:

- Amoxicilina-ácido clavulánico 500/125 mg cada $8 \mathrm{~h} \mathrm{o} \mathrm{875/125} \mathrm{mg} \mathrm{cada} 12 \mathrm{~h}$.

- Cefuroxima $500 \mathrm{mg}$ cada $12 \mathrm{~h}$. 
- Claritromicina $500 \mathrm{mg}$ cada $12 \mathrm{~h}$.

- Levofloxacina 500 mg/día.

Falla clínica o fracaso de tratamiento. Se define la falla clínica o falta de respuesta al tratamiento empírico cuando los pacientes con neumonía, luego de $72 \mathrm{~h}$ de tratamiento antibiótico, no presentan mejoría significativa de los síntomas respiratorios, persisten con fiebre $\left(\mathrm{t}^{\circ}>38^{\circ} \mathrm{C}\right)$, presentan deterioro progresivo del intercambio gaseoso con necesidad de ventilación mecánica, desarrollan shock o sepsis grave, o presentan progresión rápida de los infiltrados pulmonares en la radiografía de tórax ${ }^{30}$.

Las principales causas de falla clínica o fracaso de tratamiento son:

1. Retardo en la resolución clínica: se ha asociado con edad avanzada, alcoholismo, insuficiencia cardíaca congestiva, EPOC, NAC multilobar y la neumonía grave. En esta categoría no es necesario modificar el esquema antibiótico empírico.

2. Uso de antibióticos inapropiados.

3. Presencia de un patógeno resistente.

4. Patógeno no cubierto por el tratamiento empírico inicial.

5. Sospecha de inmunosupresión.

6. Complicaciones de la neumonía: cavitación 0 absceso pulmonar, empiema pleural, síndrome de distrés respiratorio agudo o infección extrapulmonar.

7. Causas no infecciosas: insuficiencia cardíaca descompensada, tromboembolismo pulmonar, neumonía en organización criptogénica, neumonía postobstructiva asociada a tumor, carcinoma bronquioloalveolar, hemorragia alveolar, linfoma, neumonía eosinofílica, sarcoidosis, daño pulmonar por drogas, neumonitis alérgica extrínseca, neumonitis actínica y neumonitis intersticial aguda.

Cuando el paciente evoluciona mal y se cumplen los criterios de falla clínica, se recomienda la búsqueda sistemática de las posibles causas y ampliar la cobertura antibiótica, ya que en esta categoría aumenta significativamente el riesgo de complicaciones y muerte.

\section{MEDIDAS PREVENTIVAS}

Manuel Barros $\mathrm{M}^{1}$, Claudia Cartagena $\mathrm{S}^{1}$, Luis Bavestrello $\mathrm{F}^{2}$.

Acciones comunitarias. Campañas de información en salud (evitar el hacinamiento, alcoholismo, tabaquismo, programas de vacunación, manejo de la contaminación ambiental).

Acciones en el huésped. Consejo nutricional, control de las enfermedades crónicas, tratamiento del tabaquismo y alcoholismo, vacunación de la población de riesgo.

Vacuna antineumocócica. La vacuna antineumocócica polivalente, disponible desde 1983, incluye 23 cepas de Streptococcus pneumoniae, cubriendo cerca de $90 \%$ de las cepas que ocasionan enfermedad neumocócica invasora en sujetos inmunocompetentes mayores de 5 años. La vacuna confiere protección contra la enfermedad neumocócica invasora ${ }^{31}$.

Indicaciones de la vacuna antineumocócica:

- Adultos sanos $\geq 65$ años.

- Portadores de enfermedades crónicas: cardiopatías, EPOC, nefropatías, diabetes mellitus, cirrosis hepática, pérdida crónica de LCR, asplenia funcional o anatómica, alcoholismo.

- Inmunocomprometidos, incluyendo infección por VIH, quimioterapia y neoplasias hematológicas.

Vacuna antiinfluenza. La vacuna antiinfluenza es una vacuna polivalente de virus inactivados, altamente purificada, que incluye habitualmente dos cepas de virus influenza A y una de influenza B, seleccionadas de acuerdo al perfil epidemiológico del año respectivo. Debido al cambio antigénico que se produce cada año, es necesario modificar la composición de la vacuna. La eficacia depende de múltiples factores, entre otros, la coincidencia del virus presente en la comunidad con el incluido en la vacuna, factores ambientales y factores del huésped.

Los estudios de costo-efectividad han confirmado la eficacia de la vacuna antiinfluenza en reducir la morbimortalidad asociada a la epidemia de influenza y la disminución de los gastos de salud involucrados en el manejo de los enfermos $^{32}$. Además, los estudios clínicos han confir- 
mado que la vacunación reduce el riesgo de neumonía, la hospitalización y muerte de la población senescente durante la epidemia de influenza, cuando la cepa de la vacuna es similar a la presente en la comunidad 33 .

Indicaciones de la vacuna antiinfluenza:

- Adultos sanos $\geq 65$ años.

- Portadores de enfermedades crónicas: cardiopatías, EPOC, nefropatías, diabetes mellitus,

\section{REFERENCIAS}

1. EwIG S. Community-acquired pneumonia: definition, epidemiology, and outcome. Semin Respir Infect 1999; 14: 94-102.

2. Instituto Nacional de Estadísticas. Anuarios de Estadísticas Vitales, Chile, 2000.

3. Ministerio de Salud de Chile. Departamento de Estadísticas e Información de Salud (http:// deis.minsal.cl/index.asp).

4. Niederman MS, Mandell LA, Anzueto A, Bass JB, Broughton WA, CampBell GD et al. Guidelines for the management of adults with communityacquired pneumonia. Diagnosis, assessment of severity, antimicrobial therapy, and prevention. Am J Respir Crit Care Med 2001; 163: 1730-54.

5. British Thoracic Society Standards of Care CommitTEE. British Thoracic Society guidelines for the management of community-acquired pneumonia in adults. Thorax 2001; 56 (Suppl IV): 1-64.

6. Mandell LA, Bartlett JG, Dowell SF, File TM JR, MUSHER DM, WHITNEY C. Update of practice guidelines for the management of community-acquired pneumonia in immunocompetent adults. Clin Infect Dis 2003; 37: 1405-33.

7. Sociedad Chilena de Enfermedades Respiratorias. Consenso Nacional en Neumonías Adquiridas en la Comunidad en Adultos y Niños. Rev Chil Enf Respir 1999; 15: 67-136.

8. Fine MJ, Smith MA, Carson CA, Mutha SS, Sankey SS, WeISSFeld LA et al. Prognosis and outcomes of patients with community-acquired pneumonia. A meta-analysis. JAMA 1996; 275: 134-41.

9. Guest JF, Morris A. Community-acquired pneumonia: the annual cost to the National Health Service in the United Kingdom. Eur Respir J 1997; 10: $1530-4$. cirrosis hepática, pérdida crónica de LCR, asplenia funcional o anatómica, alcoholismo.

- Embarazadas con más de 3 meses de gestación.

- Inmunocomprometidos.

- Pacientes institucionalizados (geriátricos, casas de reposo, etc.).

- Trabajadores de la salud.

- Cuidadores de sujetos con riesgo elevado.

- Viajeros a áreas geográficas de epidemia.

10. Diehr P, Wood RW, Bushyhead J, Krueger L, Wolcotт B, Tomprins RK. Prediction of pneumonia in outpatients with acute cough, a statistical approach. J Chronic Dis 1984; 37: 215-25.

11. Metlay JP, Kapoor WN, Fine MJ. Does this patient have community-acquired pneumonia? Diagnosing pneumonia by history and physical examination. JAMA 1997; 278: 1440-5.

12. Saldías F, O’Brien A, Gederlini A, Farías G, Díaz A. Neumonía adquirida en la comunidad en el anciano inmunocompetente que requiere hospitalización. Cuadro clínico, factores pronósticos y tratamiento. Arch Bronconeumol 2003; 39: 333-40.

13. Gennis P, Galiagher J, Falvo C, Baker S, Than W. Clinical criteria for the detection of pneumonia in adults: guidelines for ordering chest roentgenograms in the emergency department. J Emerg Med 1989; 7: 263-8.

14. MitTl RL JR, Schwab RJ, Duchin JS, Goin JE, Albeida SM, MiLER WT. Radiographic resolution of community-acquired pneumonia. Am J Resp Crit Care Med 1994; 149: 630-5.

15. Sanyal S, Smith PR, Saha AC, Gupta S, Berkowitz L, Homel P. Initial microbiologic studies did not affect outcome in adults hospitalized with community-acquired pneumonia. Am J Respir Crit Care Med 1999; 160: 346-8.

16. Díaz A, Calvo M, O’Brien A, Farías G, Mardónez JM, SALDíAs F. Utilidad clínica de los hemocultivos en pacientes hospitalizados por neumonía adquirida en la comunidad. Rev Méd Chile 2002; 130: 993-1000.

17. Saldías F, Mardónez JM, Marchesse M, Viviani P, Farías G, Díaz A. Neumonía adquirida en la comunidad en el adulto hospitalizado. Cuadro clínico y factores pronósticos. Rev Méd Chile 2002; 130: 1373-82. 
18. Meehan TP, Fine MJ, Krumholz HM, Scinto JD, Galusha DH, MocKaLIS JT ET AL. Quality of care, process, and outcomes in elderly patients with pneumonia. JAMA 1997; 278: 2080-4.

19. SkERRETT SJ. Diagnostic testing for communityacquired pneumonia. Clin Chest Med 1999; 20: 531-48.

20. Trucco O, Vicencio M, Salamanca L, Ojeda A, Oyonarte M, Prado V. Participación de Legionella pneumophila en neumonía extrahospitalaria del adulto en Santiago. Rev Chil Infect 1993; 10: 8995.

21. Saldías F, Mardónez JM, Marchesse M, Díaz A. Evolución clínica y pronóstico del paciente hospitalizado por neumonía adquirida en la comunidad según lugar de admisión. Rev Chile Med Intensiva 2004; 19: 13-20.

22. Riquelme R, Riquelme M, Rioseco ML, Gómez V, Gil R. Neumonía adquirida en la comunidad en Puerto Montt. Etiología. Rev Chil Enf Respir 2003; 19: A276.

23. Díaz A, Fuentes G, Couble B, Uribe R, Mercado G, Soza A ET AL. Etiología de la neumonía adquirida en la comunidad en adultos hospitalizados en Santiago, Chile: implicancias para las guías clínicas. Rev Chil Enf Respir 2005; 21: 23-32.

24. Castanheira M, Gales AC, Mendes RE, Jones RN, SADER HS. Antimicrobial susceptibility of Streptococcus pneumoniae in Latin America: results from five years of the SENTRY Antimicrobial Surveillance Program. Clin Microbiol Infect 2004; 10: 645-51.

25. Saldías F, Flores LJ, Torres C, García P, Díaz A. Susceptibilidad a antimicrobianos de Streptococcus pneumoniae en población infantil y adulta de Santiago. Período 1997-2003. Rev Méd Chile 2005; 133: 42-9.
26. Feikin DR, Schuchat A, Kolczak M, BarRett NL, HaRRISON LH, LeFrowitz L ET AL. Mortality from invasive pneumococcal pneumonia in the era of antibiotic resistance, 1995-1997. Am J Public Health 2000; 90: 223-9.

27. BJerRe LM, Verheij TJ, Kochen MM. Antibiotics for community acquired pneumonia in adult outpatients. Cochrane Database Syst Rev 2004; 2: CD002109.

28. Oosterheert JJ, Bonten MJ, HaK E, Schneider MM, HoEpeLMAN IM. How good is the evidence for the recommended empirical antimicrobial treatment of patients hospitalized because of communityacquired pneumonia? A systematic review. J Antimicrob Chemother 2003; 52: 555-63.

29. Rhew DC, Tu GS, Ofman J, Henning JM, Richards MS, WeINGARTEN SR. Early switch and early discharge strategies in patients with community-acquired pneumonia: a meta-analysis. Arch Intern Med 2001; 161: 722-7.

30. Arancibia F, Ewig S, Martínez JA, Ruiz M, Bauer T, MARcos MA ET AL. Antimicrobial treatment failures in patients with community-acquired pneumonia: causes and prognostic implications. Am J Respir Crit Care Med 2000; 162: 154-60.

31. Dear K, Holden J, Andrews R, Tatham D. Vaccines for preventing pneumococcal infection in adults. Cochrane Database Syst Rev 2003; 4: CD000422.

32. Nichol KL, Margolis KL, Wuorenma J, Von Sternberg $T$. The efficacy and cost effectiveness of vaccination against influenza among elderly persons living in the community. N Engl J Med 1994; 331: 778-84.

33. Prevention and control of influenza: recommendations of the Advisory Committee on Immunization Practices (ACIP). Centers for Disease Control and Prevention. MMWR Recomm Rep 1998; 47(RR6): 1-26. 